\title{
Investigation of Surface Plasmon Resonances in Silver Infiltrated Metalattices by Monochromated Electron Energy Loss Spectroscopy
}

Parivash Moradifar ${ }^{1}$, Yunzhi Liu ${ }^{2}$, Jennifer L. Russell ${ }^{2}$, Thomas E. Mallouk ${ }^{2}$, John Badding ${ }^{3}$ and Nasim Alem $^{1}$

1. Department of Materials Science and Engineering, Materials Research Institute, The Pennsylvania State University, University Park, PA.

2. Department of Chemistry, The Pennsylvania State University, University Park, PA.

3. Department of Physics, Department of Chemistry, Department of Materials Science and Engineering, The Pennsylvania State University, University Park, PA.

Metalattices are artificial 3D ordered periodic nanostructured solids on the range between $1 \mathrm{~nm}-100 \mathrm{~nm}$ [1-3]. A metalattice structure, can namely subdivide into meta-atoms linked through thin interconnected channels named meta-bonds. The nano-meter 3D structural order in these structures can have a profound impact on their electronic, photonic, magnetic and vibrational degrees of freedom. Although infiltrating 3D ordered frameworks, such as silica nano-opals, with semiconducting materials (such as $\mathrm{Si}$ and $\mathrm{Ge}$ ) and/or metals (such as $\mathrm{Ag}, \mathrm{Ni}$, and $\mathrm{Pt}$ ) can result in tuning their functional properties, a void-free infiltration of metalattices can be extremely challenging for a sub $100 \mathrm{~nm}$ size of frameworks. Recently, chemical fluid deposition from supercritical carbon dioxide has been shown as a promising approach to achieve void-free infiltration of such metalattice materials via penetration of supercritical fluids into the small void spaces [1].

In this study, we choose a 3D ordered interconnected system with voids in between silica opals filled with silver to create a novel structure to study the generation and propagation of plasmons in comparison to their partner structures such as isolated plasmonic nanoparticles and nanowires [4-6]. In our 3D ordered nanostructures with the metalattice size below the diffraction limit, electromagnetic radiation can be coupled with the oscillation of free charge carriers in the structure in the form of surface plasmons (SPs) [4]. Since surface plasmon phenomena at sub wavelength scales are not detectable with conventional optical microscopes, this study utilizes monochromated Scanning Transmission Electron Microscopy (STEM) and Electron Energy Loss Spectroscopy (EELS) with high spatial and energy resolution to characterize LSPRs at the nanoscale range in a broad spectral energy range [4-6]. This enables indirect mapping of electromagnetic hotspots associated with the localized surface plasmon resonances at the nanoscale, providing an in depth understanding of plasmonic behavior in metal infiltrated silica nanoopals metalattice materials. Of particular interest are silver filled silica nano-opals metalattice materials that can exhibit dark plasmon modes resulting from vanishing dipole moments, not accessible with a farfield optical measurement technique [4-6].

Figure 1a shows annular dark field STEM image of a cross-section of Ag infiltrated silica nano-opals. Spectrum maps of the separate SP peaks with their energy range appearing in the energy loss spectrum can be seen in Figure 1b-1h. As can be seen in Figure 1(f) SPs which are strictly confined to Ag infiltration of nano-opals start from meta-bonds. This work will also combine theoretical calculations to further elaborate the coupling of SP modes and interaction between confined silica environment and Ag meta bonds and meta atoms. Probing the near-field electromagnetic interactions associated with interconnected Ag-filled void spacings, including meta-bonds and meta-atoms, surrounding silica nano-opals, is a key to develop metalattice materials with enhanced photonic properties. The structural geometry, intrinsic 
materials properties, and local dielectric environment are some of the most important parameters that can alter the resulting resonant behavior of the SPs. Controlling the size/geometry of metalattice materials to produce the desired localized surface plasmon resonances (LSPRs) can lead to a more efficient electromagnetic energy storage in nanoplasmonics, enhanced biological and chemical sensing, optical data storage, and gas molecule detection [7].

\section{References:}

[1] Y.Liu et al, Nano Lett. 18(1), p. 546.

[2] J.E.Han and V.H. Crespi, Phyiscal Review Letters 86 (2001), p. 696.

[3] J.E.Han and V.H. Crespi, Phyiscal Review Letters 89 (2002), p. 197203.

[4] A.M.Thron et al, Microsc. Microanal. 20 (S3) (2014), p. 600.

[5] D. Rossouw et al, Nano Lett. 11 (2011), p. 1499.

[6] G.T.Forcherio et al, J. Phys. Chem C 120 (2016), p. 24950.

[7] This work was funded by the Penn State MRSEC, Center for Nanoscale Science, under the award NSF DMR-1420620.
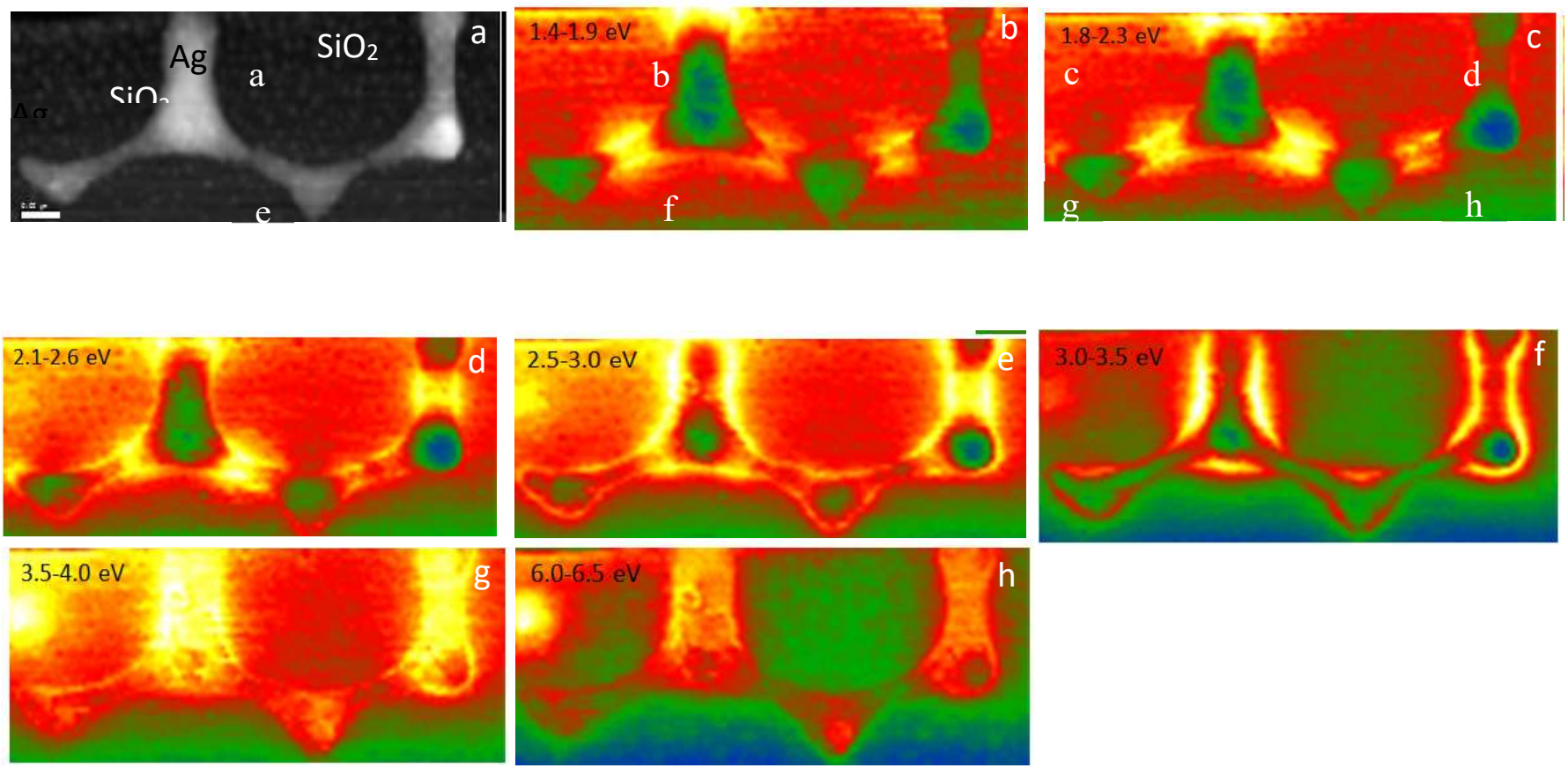

Figure 1. (a) Annular Dark Field Image of Ag filled silica nano-opals (b-g) Spectrum maps of separate SP and (h) bulk plasmon peak of Ag which appears on the spectrum. 